\title{
SOME OBSERVATIONS ON GENERALIZED SADDLE-POINT PROBLEMS*
}

\author{
P. CIARLET, JR. ${ }^{\dagger}$, JIANGUO HUANG ${ }^{\ddagger}$, AND JUN ZOU§
}

\begin{abstract}
This paper studies the solvability and stability of a generalized saddle-point system in finite- and infinite-dimensional spaces. Sharp solvability conditions and stability estimates are derived.
\end{abstract}

Key words. generalized saddle-point problems, stability, solvability

AMS subject classifications. 35A15, 35A05, 65N12, 65L20

PII. S0895479802410827

1. Introduction. We shall consider the solvability and stability of the following saddle-point system: Find $(u, p) \in V \times Q$ such that

$$
\begin{aligned}
a(u, v)+b_{1}(v, p) & =f(v) \quad \forall v \in V, \\
b_{2}(u, q)-c(p, q) & =g(q) \quad \forall q \in Q,
\end{aligned}
$$

where $a, b_{1}, b_{2}$, and $c$ are bounded bilinear forms and where $f$ and $g$ are bounded linear functionals on $V$ and $Q$, respectively. The system (1.1)-(1.2) seems to be one of the most generalized saddle-point systems investigated in the literature. The case of bilinear forms $c=0$ and $b_{1}=b_{2}$ has been extensively studied [1, 3, 4, 7, 5, 9, 10]. Also, considerable research has been done on the system with $b_{1}=b_{2}$ and $c \neq 0[4,11,14]$, while the well-posedness for the system with $c=0$ but $b_{1} \neq b_{2}$ was established in [13] and [2]. However, to our knowledge there have been no investigations into the solvability and stability for the most general form of system (1.1)-(1.2) with $b_{1} \neq b_{2}$ and $c \neq 0$.

The aim of this paper is to establish the solvability and stability conditions for the generalized saddle-point system (1.1)-(1.2). The existence and uniqueness of the solutions to the system are shown under some standard conditions, and stability estimates of the solutions are derived in terms of the given data.

The system (1.1)-(1.2) arises in, for example, mixed variational formulations of some boundary value problems. The first of such examples is the following general non-self-adjoint elliptic problem:

$$
-\nabla \cdot(\alpha(x) \nabla p+\mathbf{b}(x) p)+\gamma(x) p=\mu(x), \quad x \in \Omega,
$$

where $\Omega$ is a bounded domain in $R^{d}(d=2,3)$ with boundary $\partial \Omega$, the solution $p$ is assumed to take the boundary value $\omega(x)$ on $\partial \Omega$, and $\alpha(x), \mathbf{b}(x), \gamma(x)$, and $\mu(x)$

${ }^{*}$ Received by the editors July 10, 2002; accepted for publication (in revised form) by A. Wathen December 30, 2002; published electronically May 29, 2003.

http://www.siam.org/journals/simax/25-1/41082.html

†Ecole Nationale Supérieure de Techniques Avancées, 32, Boulevard Victor, 75739 Paris Cedex 15, France (ciarlet@ensta.fr).

$\ddagger$ Department of Mathematics, Shanghai Jiao Tong University, Shanghai, 200240, People’s Republic of China (jghuang@online.sh.cn). The work of this author was partially supported by the National Natural Science Foundation of China under grant 19901018 and by Direct Grant of CUHK (2060226), Hong Kong.

$\S$ Department of Mathematics, The Chinese University of Hong Kong, Shatin, N.T., Hong Kong (zou@math.cuhk.edu.hk). The work of this author was supported by Hong Kong RGC grants CUHK4292/00P and CUHK4048/02P. 
are given functions with appropriate smoothness [6]. By introducing the new variable $\mathbf{u}=-(\alpha \nabla p+\mathbf{b} p)$, and letting $\tilde{\alpha}(x)=\alpha(x)^{-1}$ and $\tilde{\mathbf{b}}(x)=\tilde{\alpha}(x) \mathbf{b}(x)$, we have that the weak form of (1.3) is then described by system (1.1)-(1.2) (see [6]), with two spaces $V=\left\{\mathbf{u} \in L^{2}(\Omega)^{d} ; \operatorname{div} \mathbf{v} \in L^{2}(\Omega)\right\}$ and $Q=L^{2}(\Omega)$, and two linear functionals $f(\mathbf{v})=-\langle\omega, \mathbf{v} \cdot \mathbf{n}\rangle$ and $g(q)=-(\mu, q)$, while the bilinear forms are given by

$$
\begin{array}{ll}
a(\mathbf{u}, \mathbf{v})=(\tilde{\alpha} \mathbf{u}, \mathbf{v}), & b_{1}(\mathbf{v}, p)=-(\operatorname{div} \mathbf{v}, p)+(\tilde{\mathbf{b}} p, \mathbf{v}), \\
c(p, q)=(\gamma p, q), & b_{2}(\mathbf{u}, q)=-(\operatorname{div} \mathbf{u}, q),
\end{array}
$$

where $(\cdot, \cdot)$ and $\langle\cdot, \cdot\rangle$ denote the scalar products in $L^{2}(\Omega)$ (or $\left.L^{2}(\Omega)^{d}\right)$ and $L^{2}(\partial \Omega$ ), respectively.

A second example comes from some exterior electromagnetic interface problems $[12,13]$. The weak formulations of such problems also take the form (1.1)-(1.2) if one introduces a Lagrange multiplier variable $\mathbf{u}$ for the current density $\nabla \phi$, where $\phi$ is the potential function $[12,13]$, and introduces another Lagrange multiplier variable $\xi$ for the boundary value of $\phi$ on the boundary of the physical domain $\Omega$.

2. Preliminaries. In this section, we introduce some existing saddle-point theory. Let $V$ and $Q$ be two finite- or infinite-dimensional Hilbert spaces equipped with the inner products $(\cdot, \cdot)_{V}$ and $(\cdot, \cdot)_{Q}$, and the induced norms $\|\cdot\|_{V}$ and $\|\cdot\|_{Q}$, respectively. Let $a(v, w), b_{1}(v, q)$, and $b_{2}(v, q)$ be bilinear forms on $V \times V, V \times Q$, and $V \times Q$, respectively, which are bounded; i.e., there are positive constants $\|a\|,\left\|b_{1}\right\|$, and $\left\|b_{2}\right\|$ such that

$$
\begin{array}{ll}
|a(v, w)| \leq\|a\|\|v\|_{V}\|w\|_{V} & \forall v, w \in V \\
\left|b_{1}(v, q)\right| \leq\left\|b_{1}\right\|\|v\|_{V}\|q\|_{Q} & \forall v \in V, q \in Q \\
\left|b_{2}(v, q)\right| \leq\left\|b_{2}\right\|\|v\|_{V}\|q\|_{Q} & \forall v \in V, q \in Q .
\end{array}
$$

Associated with the three bilinear forms are three linear operators $A \in \mathcal{L}(V, V), B_{1}$, $B_{2} \in \mathcal{L}(V, Q)$ defined by

$$
\begin{aligned}
& a(v, w)=(A v, w)_{V} \quad \forall v \in V, w \in V, \\
& b_{1}(v, q)=\left(B_{1} v, q\right)_{Q}=\left(v, B_{1}^{t} q\right)_{V} \quad \forall v \in V, q \in Q, \\
& b_{2}(v, q)=\left(B_{2} v, q\right)_{Q}=\left(v, B_{2}^{t} q\right)_{V} \quad \forall v \in V, q \in Q .
\end{aligned}
$$

Clearly, the three constants in (2.1)-(2.3) can be taken as

$$
\|a\|=\|A\|_{\mathcal{L}(V, V)}, \quad\left\|b_{1}\right\|=\left\|B_{1}\right\|_{\mathcal{L}(V, Q)}, \quad\left\|b_{2}\right\|=\left\|B_{2}\right\|_{\mathcal{L}(V, Q)} .
$$

We first consider the saddle-point problem

$$
\begin{aligned}
a(u, v)+b_{1}(v, p) & =(f, v)_{V} \quad \forall v \in V, \\
b_{2}(u, q) & =(g, q)_{Q} \quad \forall q \in Q .
\end{aligned}
$$

This system is equivalent to the following operator equation (or matrix equation in finite dimensions):

$$
A u+B_{1}^{t} p=f, \quad B_{2} u=g .
$$

Let $U_{i}=\operatorname{Ker}\left(B_{i}\right), i=1,2$. Then we have the following results on system (2.4) (cf. $[13,2])$. 
THEOREM 2.1. In addition to assumptions (2.1)-(2.3), we assume that

$$
\begin{aligned}
& \sup _{w \in U_{1}} \frac{a(v, w)}{\|w\|_{V}} \geq \alpha\|v\|_{V} \quad \forall v \in U_{2}, \\
& \sup _{v \in U_{2}} a(v, w)>0 \quad \forall w \in U_{1}, w \neq 0, \\
& \sup _{v \in V} \frac{b_{i}(v, q)}{\|v\|_{V}} \geq \beta_{i}\|q\|_{Q} \quad \forall q \in Q \quad(i=1,2)
\end{aligned}
$$

hold for some constants $\alpha, \beta_{1}, \beta_{2}>0$. Then for any $f \in V$ and $g \in Q$, there exists a unique solution $(u, p) \in V \times Q$ to system (2.4), and the following stability estimates hold:

$$
\begin{aligned}
\|u\|_{V} & \leq \beta_{2}^{-1}\left(1+\alpha^{-1}\|a\|\right)\|g\|_{Q}+\alpha^{-1}\|f\|_{V} \\
\|p\|_{Q} & \leq \beta_{1}^{-1}\left(\|f\|_{V}+\|a\|\|u\|_{V}\right) .
\end{aligned}
$$

Theorem 2.1 generalizes the standard saddle-point theory $\left(b_{1}=b_{2}\right)[1,3]$. Equation (2.8) is the so-called inf-sup condition, which plays an important role in the entire saddle-point theory. We refer to $[4,7]$ and the references therein for more details.

To apply the saddle-point theory for the compressible Stokes equations, Kellogg and Liu [11] introduced another abstract framework; see also [4]. Let $c(p, q)$ be a bounded and weakly coercive bilinear form on $Q \times Q$; i.e., there exist a positive constant $\|c\|$ and a constant $\gamma$ (possibly negative ${ }^{1}$ ) such that

$$
\begin{aligned}
|c(p, q)| & \leq\|c\|\|p\|_{Q}\|q\|_{Q} \quad \forall p, q \in Q \\
c(q, q) & \geq-\gamma\|q\|_{Q}^{2} \quad \forall q \in Q .
\end{aligned}
$$

Further, define the operator $C \in \mathcal{L}(Q, Q)$ by

$$
c(p, q)=(C p, q)_{Q} \quad \forall p, q \in Q .
$$

Let $b(v, q)$ be a bilinear form on $V \times Q$ satisfying

$$
\begin{aligned}
\sup _{v \in V} \frac{b(v, q)}{\|v\|_{V}} \geq \beta\|q\|_{Q} \quad \forall q \in Q, & \\
|b(v, q)| & \leq\|b\|\|v\|_{V}\|q\|_{Q} \quad \forall v \in V, q \in Q
\end{aligned}
$$

for some positive constants $\beta$ and $\|b\|$. Then for the saddle-point problem

$$
\begin{aligned}
& a(u, v)+b(v, p)=(f, v)_{V} \quad \forall v \in V, \\
& b(u, q)-c(p, q)=(g, q)_{Q} \quad \forall q \in Q,
\end{aligned}
$$

which is equivalent to the operator equation (or matrix equation)

$$
A u+B^{t} p=f, \quad B u-C p=g,
$$

we have (cf. $[11,4])$ the following.

\footnotetext{
${ }^{1}$ It is clear from (2.11) that the weak coerciveness (2.12) is always satisfied for any $\gamma \geq\|c\|$, but we are interested only in the case with $\gamma<\|c\|$.
} 
TheOrem 2.2. Assume that for some constant $\alpha>0$,

$$
a(v, v) \geq \alpha\|v\|_{V}^{2} \quad \forall v \in V,
$$

and conditions (2.1), (2.11)-(2.15) are satisfied. Then for any $f \in V$ and $g \in Q$, there exists a unique solution $(u, p) \in V \times Q$ to system (2.16) if $\gamma<\alpha\|a\|^{-2} \beta^{2}$, and the following stability estimates hold:

$$
\|p\|_{Q} \leq \frac{\alpha^{-1}\|b\|\|f\|_{V}+\|g\|_{Q}}{\alpha\|a\|^{-2} \beta^{2}-\gamma}, \quad\|u\|_{V} \leq \alpha^{-1}\left(\|f\|_{V}+\|b\|\|p\|_{Q}\right) .
$$

Finite-dimensional case. Let us briefly discuss the equivalent forms of the inf-sup condition and other conditions used in Theorems 2.1 and 2.2 when $V$ and $Q$ are finite dimensional. Without loss of generality, we consider $V=R^{n}$ and $Q=R^{m}$ $(n \geq m)$, and both spaces are equipped with the standard Euclidean norms $\|\cdot\|_{2}$ and inner products $(\cdot, \cdot)$, with no distinction between the notation of the norms and inner products of $R^{n}$ and $R^{m}$.

First, we claim that the inf-sup conditions (2.8) are equivalent to the conditions $\operatorname{rank}\left(B_{1}\right)=\operatorname{rank}\left(B_{2}\right)=m$. To see this, we write

$$
\sup _{v \in V} \frac{b_{1}(v, q)}{\|v\|_{V}}=\sup _{v \in R^{n}} \frac{\left(v, B_{1}^{t} q\right)}{\|v\|_{2}}=\left\|B_{1}^{t} q\right\|_{2}
$$

so (2.8) with $i=1$ is the same as the condition

$$
\left\|B_{1}^{t} q\right\|_{2} \geq \beta_{1}\|q\|_{2} \quad \forall q \in R^{m}
$$

or $\operatorname{rank}\left(B_{1}\right)=m$. Similar derivations lead to the fact that (2.8) with $i=2$ is equivalent to the condition $\operatorname{rank}\left(B_{2}\right)=m$.

Second, one can directly check that conditions (2.18) and (2.12) amount to

$$
\lambda_{\min }\left(\frac{A+A^{t}}{2}\right) \geq \alpha, \quad \lambda_{\min }\left(\frac{C+C^{t}}{2}\right) \geq-\gamma
$$

respectively (cf. [8]).

Finally, we analyze conditions (2.6)-(2.7). Let $\operatorname{rank}\left(B_{i}\right)=m_{i} \leq m, i=1,2$; then we know that $\operatorname{dim}\left(U_{i}\right)=\operatorname{dim}\left(\operatorname{Ker}\left(B_{i}\right)\right)=n-m_{i}$. Let $N_{i}$ be the $n \times\left(n-m_{i}\right)$ matrix formed by an orthonormal basis of $\operatorname{Ker}\left(B_{i}\right)$. To rewrite condition (2.6), for any $w \in U_{1}$ and $v \in U_{2}$, let $w=N_{1} x$ and $v=N_{2} y$ with $x \in R^{n-m_{1}}$ and $y \in R^{n-m_{2}}$; then

$$
\sup _{w \in U_{1}} \frac{a(v, w)}{\|w\|_{V}}=\sup _{x \in \mathbf{R}^{n-m_{1}}} \frac{\left(A N_{2} y, N_{1} x\right)}{\left\|N_{1} x\right\|_{2}}=\sup _{x \in \mathbf{R}^{n-m_{1}}} \frac{\left(N_{1}^{T} A N_{2} y, x\right)}{\|x\|_{2}}=\left\|N_{1}^{T} A N_{2} y\right\|_{2},
$$

so (2.6) is equivalent to the condition

$$
\left\|N_{1}^{T} A N_{2} y\right\|_{2} \geq \alpha\|y\|_{2} \quad \forall y \in R^{n-m_{2}},
$$

or $\operatorname{rank}\left(N_{1}^{T} A N_{2}\right)=n-m_{2}$. Similarly, we can rewrite condition (2.7) as

$$
\sup _{v \in U_{2}} a(v, w)=\sup _{y \in R^{n-m_{2}}}\left(A N_{2} y, N_{1} x\right)=\sup _{y \in R^{n-m_{2}}}\left(y, N_{2}^{T} A^{T} N_{1} x\right) .
$$

This indicates that (2.7) is equivalent to the condition

$$
N_{2}^{T} A^{T} N_{1} x \neq 0 \quad \forall x \neq 0,
$$

or $\operatorname{rank}\left(N_{2}^{T} A^{T} N_{1}\right)=n-m_{1}$.

One can further conclude from the above that $\operatorname{rank}\left(B_{1}\right)=m_{1}=m_{2}=\operatorname{rank}\left(B_{2}\right)$ if both conditions (2.6) and (2.7) are satisfied. 
3. Main results. This paper is concerned with the following generalized saddlepoint problem: Find $(u, p) \in V \times Q$ such that

$$
\begin{aligned}
& a(u, v)+b_{1}(v, p)=(f, v)_{V} \quad \forall v \in V, \\
& b_{2}(u, q)-c(p, q)=(g, q)_{Q} \quad \forall q \in Q .
\end{aligned}
$$

The system can be written in the operator or matrix form

$$
A u+B_{1}^{t} p=f, \quad B_{2} u-C p=g .
$$

Obviously, problem (3.1) covers systems (2.4) and (2.16) as two special cases.

In this section, we present two results on the solvability and stability for system (3.1) under two sets of different conditions: the first result requires that only one of the bilinear forms $b_{1}(v, q)$ and $b_{2}(v, q)$ satisfy the inf-sup condition; the second does not assume the weak coerciveness (2.12) for the bilinear form $c(p, q)$ with $\gamma<\alpha\|a\|^{-2} \beta^{2}$.

3.1. Well-posedness with either $b_{1}(v, q)$ or $b_{2}(v, q)$ satisfying the infsup condition. The main results of this section are summarized in the following theorem.

THEOREM 3.1. The same assumptions as in Theorem 2.2 are made but with $b(v, q)$ replaced by $b_{1}(v, q)$ here. Then for any $f \in V$ and $g \in Q$, there exists a unique solution $(u, p) \in V \times Q$ to the saddle-point problem (3.1) (or (3.2)) as long as

$$
\delta_{1}=\frac{\alpha^{-1}\left\|b_{1}\right\|\left\|b_{1}-b_{2}\right\|}{\alpha\|a\|^{-2} \beta_{1}^{2}-\gamma}<1,
$$

where $\left\|b_{1}-b_{2}\right\|=\left\|B_{1}-B_{2}\right\|_{\mathcal{L}(V, Q)}$. Further, the solution admits the stability estimates

$$
\|u\|_{V} \leq \frac{1}{1-\delta_{1}}\|\tilde{u}\|_{V}, \quad\|p\|_{Q} \leq\|\tilde{p}\|_{Q}+\frac{\left\|b_{1}-b_{2}\right\|}{\left(\alpha\|a\|^{-2} \beta_{1}^{2}-\gamma\right)\left(1-\delta_{1}\right)}\|\tilde{u}\|_{V}
$$

where $(\tilde{u}, \tilde{p})$ solves $(2.16)$ with $b$ replaced by $b_{1}$, and thus has the bounds

$$
\|\tilde{p}\|_{Q} \leq \frac{\alpha^{-1}\left\|b_{1}\right\|\|f\|_{V}+\|g\|_{Q}}{\alpha\|a\|^{-2} \beta_{1}^{2}-\gamma}, \quad\|\tilde{u}\|_{V} \leq \alpha^{-1}\left(\|f\|_{V}+\left\|b_{1}\right\|\|\tilde{p}\|_{Q}\right) .
$$

Proof. We choose $u^{0}=0 \in Q$ and determine a sequence $\left\{\left(u^{n}, p^{n}\right)\right\}$ by

$$
\begin{aligned}
& A u^{n+1}+B_{1}^{t} p^{n+1}=f, \\
& B_{1} u^{n+1}-C p^{n+1}=g+\left(B_{1}-B_{2}\right) u^{n},
\end{aligned}
$$

for $n=0,1,2, \ldots$ The sequence $\left\{\left(u^{n}, p^{n}\right)\right\}$ is well defined by Theorem 2.2. Subtracting (3.5)-(3.6) from (3.5)-(3.6) with $n$ replaced by $n-1$, it follows that

$$
\begin{aligned}
& A\left(u^{n+1}-u^{n}\right)+B_{1}^{t}\left(p^{n+1}-p^{n}\right)=0, \\
& B_{1}\left(u^{n+1}-u^{n}\right)-C\left(p^{n+1}-p^{n}\right)=\left(B_{1}-B_{2}\right)\left(u^{n}-u^{n-1}\right) .
\end{aligned}
$$

Now applying estimates (2.19) to (3.7), we have

$$
\begin{aligned}
\left\|u^{n+1}-u^{n}\right\|_{V} & \leq \alpha^{-1}\left\|b_{1}\right\|\left\|p^{n+1}-p^{n}\right\|_{Q}, \\
\left\|p^{n+1}-p^{n}\right\|_{Q} & \leq \frac{\left\|b_{1}-b_{2}\right\|}{\alpha\|a\|^{-2} \beta_{1}^{2}-\gamma}\left\|u^{n}-u^{n-1}\right\|_{V},
\end{aligned}
$$


which implies for $n \geq 1$ that

$$
\left\|u^{n+1}-u^{n}\right\|_{V} \leq \delta_{1}\left\|u^{n}-u^{n-1}\right\|_{V} \leq \delta_{1}^{n}\left\|u^{1}\right\|_{V}
$$

that is, for any nonnegative integers $m>n$,

$$
\begin{aligned}
\left\|u^{m}-u^{n}\right\|_{V} & \leq \sum_{i=n}^{m-1}\left\|u^{i+1}-u^{i}\right\|_{V} \leq\left(\sum_{i=n}^{m-1} \delta_{1}^{i}\right)\left\|u^{1}\right\|_{V} \\
& \leq \frac{\delta_{1}^{n}}{1-\delta_{1}}\left\|u^{1}\right\|_{V} .
\end{aligned}
$$

This means $\left\{u^{n}\right\}$ is a Cauchy sequence, and there exists a $u \in V$ such that

$$
u^{n} \rightarrow u \quad \text { in } V \text {. }
$$

On the other hand, it follows from (3.9) and (3.10) that

$$
\left\|p^{n+1}-p^{n}\right\|_{Q} \leq \frac{\left\|b_{1}-b_{2}\right\|}{\alpha\|a\|^{-2} \beta_{1}^{2}-\gamma} \delta_{1}^{n-1}\left\|u^{1}\right\|_{V}
$$

which implies that

$$
\left\|p^{m}-p^{n}\right\|_{Q} \leq \frac{\left\|b_{1}-b_{2}\right\|}{\alpha\|a\|^{-2} \beta_{1}^{2}-\gamma} \frac{\delta_{1}^{n-1}}{1-\delta_{1}}\left\|u^{1}\right\|_{V} .
$$

Hence $\left\{p^{n}\right\}$ also is a Cauchy sequence, and there exists a $p \in Q$ such that

$$
p^{n} \rightarrow p \quad \text { in } Q .
$$

Letting $n$ tend to infinity in (3.5)-(3.6), we know that $(u, p) \in V \times Q$ solves (3.2).

We next verify the uniqueness of problem (3.2). Assume that there are two solutions $\left(u_{1}, p_{1}\right),\left(u_{2}, p_{2}\right) \in V \times Q$ to the system. It is easy to see that the difference between the two solutions satisfies

$$
\begin{aligned}
& A\left(u_{1}-u_{2}\right)+B_{1}^{t}\left(p_{1}-p_{2}\right)=0, \\
& B_{1}\left(u_{1}-u_{2}\right)-C\left(p_{1}-p_{2}\right)=\left(B_{1}-B_{2}\right)\left(u_{1}-u_{2}\right) .
\end{aligned}
$$

Using the same technique for deriving estimate (3.10), we have

$$
\left\|u_{1}-u_{2}\right\|_{V} \leq \delta_{1}\left\|u_{1}-u_{2}\right\|_{V}
$$

which shows $u_{1}=u_{2}$ since $\delta_{1}<1$. Equality $p_{1}=p_{2}$ follows immediately by applying estimate (2.19) to (3.15).

Finally, we derive the stability estimates. As $u^{0}=0$, we see that $\left(u^{1}, p^{1}\right)$ solves (2.16) with $b$ replaced by $b_{1}$; thus $\left(u^{1}, p^{1}\right)$ satisfies estimates (2.19). Taking $n=1$ in (3.11) and letting $m$ go to infinity, we obtain the first estimate in (3.4). Similarly, taking $n=1$ in (3.13) leads to the second estimate in (3.4).

Sharpness of the condition on $\delta_{1}$ in (3.3). Below, we give a simple example to show that condition $\delta_{1}<1$ is a sharp condition guaranteeing the unique solvability of system (3.2). For this, consider $V=R^{n}, Q=R^{m}$, where $n \geq m$. We choose $A=I_{n}, C=I_{m}$, and $B_{2}=-B_{1}$ with $B_{1} \in R^{m \times n}$ such that $\operatorname{rank}\left(B_{1}\right)=m$. It is easy 
to see that $\delta_{1}=2 \sigma_{\max }^{2}\left(B_{1}\right) /\left(1+\sigma_{\min }^{2}\left(B_{1}\right)\right)$, where $\sigma_{\min }\left(B_{1}\right)$ is the minimal singular value of $B_{1}$. Then $\delta_{1}<1$ means that

$$
2 \sigma_{\max }^{2}\left(B_{1}\right)<1+\sigma_{\min }^{2}\left(B_{1}\right)
$$

which implies $\sigma_{\max }\left(B_{1}\right)<1$. It is also easy to show that problem (3.2) is uniquely solvable if and only if the matrix $\left(I_{m}-B_{1} B_{1}^{t}\right)$ is nonsingular. Hence, $\sigma_{\max }\left(B_{1}\right)<1$ is indeed a sufficient condition for the unique solvability of (3.2).

On the other hand, for any $\delta_{1} \geq 1$, choose the $m \times n$ matrices $B_{1}$ and $B_{2}$ as follows:

$$
-B_{2}=B_{1}=\left(\begin{array}{ccc}
1 & 0 & 0 \\
0 & \delta_{1} I_{m-1} & 0
\end{array}\right) .
$$

Then the matrix $\left(I_{m}-B_{1} B_{1}^{t}\right)$ is singular, and so (3.2) is not uniquely solvable.

Remark 3.1. In most applications, the constants $\gamma$ in (2.12) are negative. Then the condition $\gamma \leq \alpha\|a\|^{-2} \beta_{1}^{2}$ required in Theorems 2.2 and 3.1 is automatically satisfied.

Remark 3.2. In Theorem 3.1, only $b_{1}(v, q)$, not $b_{2}(v, q)$, is required to satisfy the inf-sup condition. Similar results hold when $b_{2}(v, q)$ satisfies the inf-sup condition but $b_{1}(v, q)$ does not.

3.2. Well-posedness not assuming condition (2.12) for any $\gamma<\|c\|$. The main results of this section are summarized in the following theorem.

THEOREM 3.2. If we make the same assumptions as in Theorem 2.1, then for any $f \in V$ and $g \in Q$, there exists a unique solution $(u, p) \in V \times Q$ to the saddle-point problem (3.1) as long as

$$
\delta_{2}:=\beta_{1}^{-1} \beta_{2}^{-1}\|a\|\left(1+\alpha^{-1}\|a\|\right)\|c\|<1 .
$$

Further, the following stability estimates hold:

$$
\|p\|_{Q} \leq \frac{1}{1-\delta_{2}}\|\tilde{p}\|_{Q}, \quad\|u\|_{V} \leq\|\tilde{u}\|_{V}+\frac{\beta_{2}\left(1+\alpha^{-1}\|a\|\right)\|c\|}{1-\delta_{2}}\|\tilde{p}\|_{Q}
$$

where $(\tilde{u}, \tilde{p})$ is the solution to (2.4) and thus has the bounds

$$
\|\tilde{u}\|_{V} \leq \beta_{2}^{-1}\left(1+\alpha^{-1}\|a\|\right)\|g\|_{Q}+\alpha^{-1}\|f\|_{V}, \quad\|\tilde{p}\|_{Q} \leq \beta_{1}^{-1}\left(\|f\|_{V}+\|a\|\|\tilde{u}\|_{V}\right) .
$$

Proof. We first prove the existence of the solution to system (3.2), which is equivalent to (3.1). Choose $p^{0}=0 \in Q$, then determine a sequence $\left\{\left(u^{n}, p^{n}\right)\right\}$ by

$$
\begin{aligned}
A u^{n+1}+B_{1}^{t} p^{n+1} & =f, \\
B_{2} u^{n+1} & =g+C p^{n}
\end{aligned}
$$

for $n=0,1,2, \ldots$ By Theorem 2.1, $\left\{\left(u^{n}, p^{n}\right)\right\}$ is well defined. From (3.19)-(3.20) we have

$$
\begin{aligned}
A\left(u^{n+1}-u^{n}\right)+B_{1}^{t}\left(p^{n+1}-p^{n}\right) & =0, \\
B_{2}\left(u^{n+1}-u^{n}\right) & =C\left(p^{n}-p^{n-1}\right) .
\end{aligned}
$$

Applying estimates (2.9)-(2.10) to this system, we obtain

$$
\begin{aligned}
\left\|u^{n+1}-u^{n}\right\|_{V} & \leq \beta_{2}^{-1}\left(1+\alpha^{-1}\|a\|\right)\|c\|\left\|p^{n}-p^{n-1}\right\|_{Q}, \\
\left\|p^{n+1}-p^{n}\right\|_{Q} & \leq \beta_{1}^{-1}\|a\|\left\|u^{n+1}-u^{n}\right\|_{V},
\end{aligned}
$$


which implies for $n \geq 1$,

$$
\left\|p^{n+1}-p^{n}\right\|_{Q} \leq \delta_{2}\left\|p^{n}-p^{n-1}\right\|_{Q} \leq \delta_{2}^{n}\left\|p^{1}\right\|_{Q} .
$$

Therefore, for any nonnegative integer $m>n$,

$$
\begin{aligned}
\left\|p^{m}-p^{n}\right\|_{Q} & \leq \sum_{i=n}^{m-1}\left\|p^{i+1}-p^{i}\right\|_{Q} \leq\left(\sum_{i=n}^{m-1} \delta_{2}^{i}\right)\left\|p^{1}\right\|_{Q} \\
& \leq \frac{\delta_{2}^{n}}{1-\delta_{2}}\left\|p^{1}\right\|_{Q} .
\end{aligned}
$$

That is, $\left\{p^{n}\right\}$ is a Cauchy sequence, and there exists a $p \in Q$ such that

$$
p^{n} \rightarrow p \quad \text { in } Q .
$$

On the other hand, it follows from (3.23) and (3.25) that

$$
\left\|u^{n+1}-u^{n}\right\|_{V} \leq \beta_{2}^{-1}\left(1+\alpha^{-1}\|a\|\right)\|c\| \delta_{2}^{n-1}\left\|p^{1}\right\|_{Q},
$$

which implies that for any integer $m>n$,

$$
\left\|u^{m}-u^{n}\right\|_{V} \leq \beta_{2}^{-1}\left(1+\alpha^{-1}\|a\|\right)\|c\| \frac{\delta_{2}^{n-1}}{1-\delta_{2}}\left\|p^{1}\right\|_{Q} .
$$

Hence $\left\{u^{n}\right\}$ is also a Cauchy sequence, and there exists a $u \in V$ such that

$$
u^{n} \rightarrow u \quad \text { in } V .
$$

Letting $n$ tend to infinity in (3.19)-(3.20), we see that $(u, p) \in V \times Q$ solves (3.2). The uniqueness of the solution can be shown using an argument similar to the one used in Theorem 3.1.

It remains to give stability estimates (3.18). As $p^{0}=0$, we see from (3.19)-(3.20) that $\left(u^{1}, p^{1}\right)$ solves systems $(2.4)-(2.5)$. Then taking $n=1$ in the estimate (3.26) gives

$$
\left\|p^{m}\right\|_{Q} \leq \frac{1}{1-\delta_{2}}\left\|p^{1}\right\|_{Q},
$$

which leads to the first estimate in (3.18) by letting $m$ tend to infinity with the help of estimates (2.9)-(2.10) for $\left(u^{1}, p^{1}\right)$. In the same manner, taking $n=1$ in (3.28) leads to the second estimate in (3.18).

Sharpness of the condition on $\delta_{2}$ in (3.17). Next, we give some simple examples to show that condition (3.17) is a sharp condition guaranteeing the unique solvability of system (3.2). Clearly, in the finite-dimensional case, we have

$$
\delta_{2}=\beta_{1}^{-1} \beta_{2}^{-1}\|A\|_{2}\left(1+\alpha^{-1}\|A\|_{2}\right)\|C\|_{2} .
$$

Our first example shows that system (3.2) may not necessarily be uniquely solvable if $\delta_{2}=1$. For this, consider $V=R^{3}$ equipped with the Euclidean norm, $Q=R^{1}$ in (3.1) or (3.2). Then we choose $B_{1}=(1,0,0), B_{2}=(k, 0,0), C=-1$, with $k$ a nonzero 
constant to be determined later. For the matrix $A$, we take the following symmetric form with $\varepsilon>0$ :

$$
A=\left(\begin{array}{ccc}
a_{11} & a_{12} & \varepsilon \\
a_{12} & \varepsilon & 0 \\
\varepsilon & 0 & \varepsilon
\end{array}\right) .
$$

One can easily verify that conditions (2.6)-(2.7) hold with $\alpha=\varepsilon, \beta_{1}=1$, and $\beta_{2}=|k|$.

The next steps are intended to construct the matrix $A$ and constant $k$ such that $\delta_{2}=1$, but system (3.2) is not uniquely solvable. That is,

$$
|k|=a\left(1+\varepsilon^{-1} a\right), \quad a=\|A\|_{2},
$$

and

$$
\operatorname{det}\left(\begin{array}{cc}
A & B_{1}^{t} \\
B_{2} & -C
\end{array}\right)=\operatorname{det} A-k \varepsilon^{2}=0 .
$$

To do this construction, we want to be able to choose the matrix $A$ with three eigenvalues $a, a_{1}$, and $-a_{1}$, respectively, with $a_{1}>0$. In this case, we obtain from (3.31) and (3.32) that

$$
a a_{1}^{2}=|\operatorname{det} A|=|k| \varepsilon^{2}=a\left(1+\varepsilon^{-1} a\right) \varepsilon^{2},
$$

which gives

$$
a_{1}=\sqrt{\varepsilon^{2}+a \varepsilon}
$$

As $a=\|A\|_{2}$, we must have $a_{1} \leq a$, i.e.,

$$
\varepsilon^{2}+a \varepsilon \leq a^{2} .
$$

On the other hand, the characteristic equation of $A$ is

$$
\lambda^{3}-d_{1} \lambda^{2}+d_{2} \lambda-\operatorname{det} A=0,
$$

with $d_{1}=a_{11}+2 \varepsilon$ and $d_{2}=2 a_{11} \varepsilon-a_{12}^{2}$. Then by the Vita theorem we know that

$$
\begin{aligned}
a+a_{1}+\left(-a_{1}\right) & =a_{11}+2 \varepsilon, \\
a a_{1}+a\left(-a_{1}\right)+\left(-a_{1}^{2}\right) & =2 a_{11} \varepsilon-a_{12}^{2} .
\end{aligned}
$$

From (3.36) we get

$$
a_{11}=a-2 \varepsilon .
$$

Combining this with (3.34) and (3.37) leads to

$$
a_{12}^{2}=2 a_{11} \varepsilon+\left(\varepsilon^{2}+a \varepsilon\right)=3 \varepsilon(a-\varepsilon) .
$$

Then if we take

$$
\varepsilon \leq a / 2,
$$

(3.35) is satisfied, and by (3.39) we may choose

$$
a_{12}= \pm \sqrt{3 \varepsilon(a-\varepsilon)} .
$$


In summary, for any fixed constant $a>0$, we may choose $\varepsilon \in(0, a / 2]$, then compute $a_{11}$ and $a_{12}$ from (3.38) and (3.41), and $k$ from (3.31). Clearly, with the matrix $A$ constructed above, we have $\delta_{2}=1,\|A\|_{2}=a$, and $|\operatorname{det} A|=|k| \varepsilon^{2}$ (see (3.33)).

Using $|\operatorname{det} A|=|k| \varepsilon^{2}$, we have either $\operatorname{det} A=k \varepsilon^{2}$ or $\operatorname{det} A=-k \varepsilon^{2}$. If the former is valid, then (3.32) holds, and system (3.2) is singular; otherwise we should choose $B_{2}=-k B_{1}$. Then (3.32) holds with $k$ replaced by $-k$, and (3.2) is again singular.

Our second example shows some very interesting results when $V=R^{2}$ and $Q=$ $R^{1}$ : system (3.2) is always uniquely solvable when $\delta_{2}=1$ but may not be when $\delta_{2}>1$.

To see this, we take $A=\left(\begin{array}{cc}a_{11} & a_{12} \\ a_{21} & a_{22}\end{array}\right), C=-1, B_{1}=(1,0)$, and $B_{2}=(k, k)$. In this case, system (3.2) reads as follows:

$$
\left(\begin{array}{ccc}
a_{11} & a_{12} & 1 \\
a_{21} & a_{22} & 0 \\
k & k & 1
\end{array}\right)\left(\begin{array}{c}
u_{1} \\
u_{2} \\
p
\end{array}\right)=\left(\begin{array}{c}
f_{1} \\
f_{2} \\
g
\end{array}\right) .
$$

Clearly, both $B_{1}$ and $B_{2}$ satisfy the inf-sup conditions (2.8) with $\beta_{1}=1$ and $\beta_{2}=$ $\sqrt{2}|k|$, respectively, and $U_{1}=\operatorname{span}\left\{(0,1)^{t}\right\}$ and $U_{2}=\operatorname{span}\left\{(1,-1)^{t}\right\}$. For condition (2.6), a simple calculation gives

$$
\inf _{v \in U_{2}} \sup _{w \in U_{1}} \frac{(A v, w)}{\|v\|_{2}\|w\|_{2}}=\frac{\left|a_{21}-a_{22}\right|}{\sqrt{2}}
$$

thus (2.6) holds with $\alpha=\left|a_{21}-a_{22}\right| / \sqrt{2}$, and we should assume $a_{21} \neq a_{22}$. The condition $a_{21} \neq a_{22}$ also ensures condition (2.7). Furthermore, it follows from the definition of (3.30) that

$$
\sqrt{2}|k|=\frac{1}{\delta_{2}} a\left(1+\alpha^{-1} a\right)
$$

where $a=\|A\|_{2}=\sigma_{\max }(A)$ stands for the maximal singular value of $A$. On the other hand, problem (3.42) is uniquely solvable if and only if its coefficient matrix is nonsingular, namely,

$$
\operatorname{det}\left(\begin{array}{ccc}
a_{11} & a_{12} & 1 \\
a_{21} & a_{22} & 0 \\
k & k & 1
\end{array}\right)=k\left(a_{21}-a_{22}\right)+\operatorname{det} A \neq 0 .
$$

Let $a_{1}$ be the other singular value of $A$. Then $a^{2}$ and $a_{1}^{2}$ are the two eigenvalues of $A^{t} A$, and we have

$$
a^{2}+a_{1}^{2}=\operatorname{tr}\left(A^{t} A\right), \quad a^{2} a_{1}^{2}=|\operatorname{det} A|^{2} .
$$

Let us first consider any given $\delta_{2}>1$. We want to be able to find a constant $k>0$ and a matrix $A$ such that both (3.44) and

$$
k\left(a_{21}-a_{22}\right)+\operatorname{det} A=0
$$

hold. That is, it is possible to construct an example of the linear system (3.42), which is not uniquely solvable.

It follows from (3.43), (3.44), and (3.47) that

$$
|\operatorname{det} A|=|k|\left|a_{21}-a_{22}\right|=\sqrt{2}|k| \alpha=\frac{1}{\delta_{2}} \alpha a\left(1+\alpha^{-1} a\right)=\frac{1}{\delta_{2}} a(\alpha+a) .
$$


Combining this with the second equation of (3.46), we see that

$$
a^{2} a_{1}^{2}=\left(\frac{1}{\delta_{2}} a(\alpha+a)\right)^{2}
$$

or $a_{1}=(a+\alpha) / \delta_{2}$. To ensure $a=\|A\|_{2}$ we need $a_{1} \leq a$, that is,

$$
a \geq \frac{\alpha}{\delta_{2}-1} .
$$

Now we have to check the first equation of (3.46), that is,

$$
a^{2}+a_{1}^{2}=\operatorname{tr}\left(A^{t} A\right)=a_{11}^{2}+a_{22}^{2}+a_{12}^{2}+a_{21}^{2} .
$$

For simplicity, we take $a_{22}=0$. Then from definition (3.43), we have $a_{21}=\sqrt{2} \alpha$ (or $-\sqrt{2} \alpha$ ), so the condition $a_{21} \neq a_{22}$ is fulfilled. Now we take $a_{12}=a_{21}=\sqrt{2} \alpha$, and (3.50) becomes

$$
a^{2}+a_{1}^{2}=a_{11}^{2}+4 \alpha^{2}
$$

which gives

$$
a_{11}= \pm \sqrt{a^{2}+a_{1}^{2}-4 \alpha^{2}}
$$

if $a \geq 2 \alpha$. Therefore, given $\alpha>0$, if $a$ satisfies the condition

$$
a \geq \alpha \max \left\{2, \frac{1}{\delta_{2}-1}\right\}
$$

we obtain a suitable matrix $A$ by the above construction.

Hence, for any fixed $\alpha>0$, we can choose $a>0$ satisfying condition (3.51), and afterwards choose $k$ from (3.44). Then we can compute $A$ from (3.50)-(3.51). Clearly, with such a resulting matrix $A$, we have $\delta_{2}=1,\|A\|_{2}=a$, and $|\operatorname{det} A|=\left|k a_{21}\right|$ (see (3.48)).

As $|\operatorname{det} A|=\left|k a_{21}\right|$, we have either $\operatorname{det} A=-k a_{21}$ or $\operatorname{det} A=k a_{21}$. If the former is valid, then (3.47) holds, and system (3.42) is singular. Otherwise we should choose $B_{2}=-(k, k)$; then (3.47) is satisfied with $k$ replaced by $-k$, and (3.42) is again singular.

Finally, we consider the case $\delta_{2}=1$. To our surprise, this condition guarantees the unique solvability of (3.42) when $V=R^{2}$ and $Q=R^{1}$. This is summarized in the next proposition.

Proposition 3.3. Let $V=R^{2}, Q=R^{1}$ and $A, B_{1}$, and $B_{2}$ satisfy conditions (2.6)-(2.8), $C \neq 0$. Then for any $f \in R^{2}$ and $g \in R^{1}$, problem (3.2) is uniquely solvable.

Proof. Without loss of generality, assume $C=-1$. We proceed by contradiction. Assume that there exist $A=\left(\begin{array}{cc}a_{11} & a_{12} \\ a_{21} & a_{22}\end{array}\right), B_{1}=\left(b_{1}, b_{2}\right), B_{2}=\left(c_{1}, c_{2}\right)$ such that $\delta_{2}=1$; however, system (3.2) is singular, that is,

$$
\operatorname{det}\left(\begin{array}{ccc}
a_{11} & a_{12} & b_{1} \\
a_{21} & a_{22} & b_{2} \\
c_{1} & c_{2} & 1
\end{array}\right)=0 .
$$


Clearly, $\operatorname{Ker}\left(B_{1}\right)=\operatorname{span}\left\{\left(-b_{2}, b_{1}\right)^{t}\right\}, \operatorname{Ker}\left(B_{2}\right)=\operatorname{span}\left\{\left(-c_{2}, c_{1}\right)^{t}\right\}, \beta_{1}=\left\|B_{1}\right\|_{2}$, $\beta_{2}=\left\|B_{2}\right\|_{2}$, and conditions (2.6)-(2.7) are equivalent to

$$
\frac{\left|\left(-b_{2}, b_{1}\right)\left(\begin{array}{ll}
a_{11} & a_{12} \\
a_{21} & a_{22}
\end{array}\right)\left(\begin{array}{r}
-c_{2} \\
c_{1}
\end{array}\right)\right|}{\left\|B_{1}\right\|_{2}\left\|B_{2}\right\|_{2}}=\frac{|\operatorname{det} A|}{\left\|B_{1}\right\|_{2}\left\|B_{2}\right\|_{2}}=\alpha>0,
$$

where we have used the fact, thanks to (3.52), that there holds

$$
\operatorname{det} A=b_{2} c_{2} a_{11}-b_{1} c_{2} a_{21}-b_{2} c_{1} a_{12}+b_{1} c_{1} a_{22} .
$$

On the other hand, it follows from (3.30) and $\delta_{2}=1$ that

$$
\left\|B_{1}\right\|_{2}\left\|B_{2}\right\|_{2}=\|A\|_{2}\left(1+\alpha^{-1}\|A\|_{2}\right)
$$

This with (3.53) implies

$$
|\operatorname{det} A|=\left\|B_{1}\right\|_{2}\left\|B_{2}\right\|_{2} \alpha=\|A\|_{2}\left(\|A\|_{2}+\alpha\right) .
$$

Let $\sigma_{1}$ be the smallest singular value of $A$ in comparison with the singular value $a=\|A\|_{2}$; then from (3.46) and (3.55) it follows that

$$
\|A\|_{2}^{2} \sigma_{1}^{2}=|\operatorname{det} A|^{2}=\|A\|_{2}^{2}\left(\|A\|_{2}+\alpha\right)^{2},
$$

which gives $\sigma_{1}=\|A\|_{2}+\alpha$. This is a contradiction.

Concluding remarks. We have studied the solvability and stability of a generalized saddle-point system in finite- or infinite-dimensional spaces. Sharp solvability conditions and stability estimates are derived. The results generalize some existing saddle-point theories in such a natural way that the results here reduce to the existing ones in the special cases. For example, Theorem 3.1 reduces to Theorem 2.2 when two bilinear forms $b_{1}$ and $b_{2}$ are equal, while Theorem 3.2 reduces to Theorem 2.1 when the bilinear form $c(p, q)$ vanishes.

Theorems 3.1 and 3.2 hold for both finite- and infinite-dimensional Hilbert spaces $V$ and $Q$. In the case that $V$ and $Q$ are infinite dimensional, one may further consider their finite-dimensional approximations $V_{h}$ and $Q_{h}$, e.g., by finite element methods, and establish the error estimates for the approximate solutions of problem (3.1) associated with the spaces $V_{h}$ and $Q_{h}$. The detailed discussions on the error estimates are omitted here as they follow naturally from the standard error estimates for systems (2.4) and $(2.16)$, as done in $[13,4]$. For the solvability and stability of the resulting finite-dimensional system, one of the most important and difficult issues is to appropriately choose the pair $\left(V_{h}, Q_{h}\right)$ such that the inf-sup conditions are held with the constants $\beta_{i}$ in (2.8) and $\beta$ in (2.14) independent of the mesh parameter $h$.

Acknowledgments. The authors wish to thank two anonymous referees for many constructive comments.

\section{REFERENCES}

[1] I. Babuska And A. AzIz, Survey lectures on the mathematical foundations of the finite element method, in The Mathematical Foundations of the Finite Element Method with Applications to Partial Differential Equations, A. Aziz, ed., Academic Press, New York, 1973, pp. 1-359.

[2] C. Bernardi, C. Canuto, and Y. Maday, Generalized inf-sup conditions for Chebyshev spectral approximation of the Stokes problem, SIAM J. Numer. Anal., 25 (1988), pp. 1237-1271. 
[3] F. BrezzI, On the existence, uniqueness and approximation of saddle point problems arising from Lagrange multipliers, RAIRO Math. Model. Numer. Anal., 8 (1974), pp. 129-151.

[4] F. Brezzi And M. Fortin, Mixed and Hybrid Element Methods, Springer-Verlag, New York, 1991.

[5] P. Ciarlet, JR. and J. Zou, Finite element convergence for the Darwin model to Maxwell's equations, RAIRO Math. Model. Numer. Anal., 31 (1997), pp. 213-250.

[6] J. Douglas, JR. And J. Roberts, Global estimates for mixed methods for second order elliptic equations, Math. Comput., 44 (1985), pp. 39-52.

[7] V. Girault and P. Raviart, Finite Element Methods for Navier-Stokes Equations, SpringerVerlag, New York, 1986.

[8] G. H. Golub and C. F. Van Loan, Matrix Computation, 2nd ed., Johns Hopkins University Press, Baltimore, 1989.

[9] Q. Hu AND J. Zou, An iterative method with variable relaxation parameters for saddle-point problems, SIAM J. Matrix Anal. Appl., 23 (2001), pp. 317-338.

[10] Q. Hu AND J. ZoU, Two new variants of nonlinear inexact Uzawa algorithms for saddle-point problems, Numer. Math., 93 (2002), pp. 333-359.

[11] R. B. KellogG And B. Liu, A finite element method for compressible Stokes equations, SIAM J. Numer. Anal., 33 (1996), pp. 780-788.

[12] R. MacCamy, Variational procedures for a class of exterior interface problems, J. Math. Anal. Appl., 78 (1980), pp. 248-266.

[13] R. A. Nicolaides, Existence, uniqueness and approximation for generalized saddle point problems, SIAM J. Numer. Anal., 19 (1982), pp. 349-357.

[14] D. YANG, Iterative schemes for mixed finite element methods with applications to elasticity and compressible flow problems, Numer. Math., 93 (2002), pp. 177-200. 